\title{
BMJ Open Polymyxin B-immobilised haemoperfusion and mortality in critically ill patients with sepsis/septic shock: a protocol for a systematic review and meta-analysis
}

\author{
Tomoko Fujii, ${ }^{1,2}$ Riki Ganeko, ${ }^{3}$ Yuki Kataoka, ${ }^{4}$ Robin Featherstone, ${ }^{5}$ \\ Sean M Bagshaw, ${ }^{6}$ Toshi A Furukawa ${ }^{7}$
}

To cite: Fujii T, Ganeko R, Kataoka $Y$, et al. Polymyxin B-immobilised haemoperfusion and mortality in critically ill patients with sepsis/septic shock: a protocol for a systematic review and metaanalysis. BMJ Open 2016;6: e012908. doi:10.1136/ bmjopen-2016-012908

- Prepublication history and additional material is available. To view please visit the journal (http://dx.doi.org/ 10.1136/bmjopen-2016012908).

Received 31 May 2016 Revised 5 September 2016 Accepted 4 October 2016

CrossMark

For numbered affiliations see end of article.

Correspondence to

Professor Toshi A Furukawa; furukawa@kuhp.kyoto-u.ac.jp

\section{ABSTRACT}

Introduction: Polymyxin-B immobilised haemoperfusion (PMX-HP) is a promising adjuvant strategy for the treatment of sepsis and septic shock. PMX-HP therapy works by clearing circulating endotoxin through binding to polymyxin-immobilised fibres during haemoperfusion. Small clinical trials have shown that PMX-HP therapy is associated with improved haemodynamic profile, oxygenation and survival. However, clear inferences have been largely inconclusive due to limitations in study design (eg, small, unblinded) and generalisability. We therefore propose to perform an up-to-date systematic review and evidence synthesis to describe the efficacy, safety and effectiveness of PMX-HP for adult patients with sepsis or septic shock.

Methods and analysis: We will search the following databases from 1946 to 2016 MEDLINE (Ovid), EMBASE (Ovid), Cochrane Library, Health Technology Assessment Database (HTA), Cumulative Index to Nursing and Allied Health Literature (CINAHL), PubMed and 'Igaku Chuo Zasshi' (ICHUSHI) for randomised controlled trials of PMX-HP in critically ill patients with sepsis or septic shock. There will be no language restrictions in the electronic search for studies. Two reviewers will extract data and appraise the quality of each study independently. The primary outcome will be the pooled risk ratio of 28-day all-cause mortality. Serious adverse events and changes in organ dysfunction scores will also be evaluated. The secondary outcomes will be 90-day all-cause mortality, changes in haemodynamic profile and endotoxin levels, and health services use.

Ethics and dissemination: Our systematic review will synthesise the evidence on use of the PMX-HP as an adjuvant therapy in sepsis/septic shock to improve patient-centred, physiological and health services outcomes. Research ethics is not required for this review. The study will be disseminated by peer-reviewed publication and conference presentation.

Trial registration number: CRD42016038356.
Strengths and limitations of this study

- This is a protocol for a systematic review and evidence synthesis of randomised trials evaluating the impact of polymyxin-B immobilised haemoperfusion (PMX-HP) on outcomes in sepsis/ septic shock.

- We have assembled a strong interprofessional team to perform this systematic review that includes clinical content experts, methodology experts and a research librarian experienced in the performance of high-quality systematic reviews.

- We have developed a rigorous peer-reviewed high-yield literature search strategy to identify relevant randomised trial for inclusion.

- The search results may yield numerous small clinical trials with significant clinical and statistical heterogeneity that may present challenges for interpretation.

- This systematic review is an important initial step in the assessment of PMX-HP technology that will inform clinical practice guidelines for recommendations on its usage as an adjuvant therapy of sepsis/septic shock in the clinical practice guidelines.

\section{INTRODUCTION}

Description of the condition

Sepsis remains desperately fatal and is a major cause of mortality in the intensive care unit (ICU) settings worldwide. ${ }^{1}{ }^{2}$ Many efforts have been made to improve prognosis in this condition but large multicentred trials of various innovative therapies have failed to demonstrate consistent benefit. ${ }^{3}$ Since basic elements of sepsis treatment, including definitive antibiotic therapy, adequate fluids and vasopressors, have not changed for decades, ${ }^{4}{ }_{5}$ new effective therapies are desperately needed. 


\section{Description of the intervention}

Experimental animal models of peritonitis have been used to understand the pathophysiological mechanisms involved in sepsis and septic shock. Among these mechanisms, endotoxin, one of the components on the outer membrane of gram-negative bacteria, is recognised as a potent mediator of the host response in sepsis. A study measuring endotoxin levels in patients with septic shock found that high levels of endotoxin activity were associated with worse clinical outcomes. ${ }^{6}$

Polymyxin (PMX) is a cyclic cationic polypeptide antibiotic with high affinity for endotoxin. A novel strategy whereby PMX is bound and immobilised to polystyrene fibres in a haemoperfusion device was developed in Japan. $^{78}$

\section{How the intervention might work}

The suggested mechanism of action of PMX haemoperfusion (PMX-HP) is to remove circulating endotoxin by adsorption, which limits and disrupts the maladaptive host response to infection and the progression of the biological cascade of sepsis.

\section{Why it is important to do this review}

The role of endotoxin in sepsis is well established in the literature. $^{9} 10$ Some clinical trials have shown that PMX-HP can improve the physiological profile of patients with abdominal sepsis. ${ }^{11}{ }^{12}$ In a small pilot study, cardiac index and oxygen delivery index were improved in patients treated with PMX-HP. ${ }^{11}$ In a multicentre Italian trial, Cruz et $a l^{12}$ showed that PMX-HP improved blood pressure and decreased dose requirement for vasopressor support. However, it has not been determined whether they offer any clinically important benefits and actually translate into improved outcomes for patients. Individual studies tend to focus on surrogate outcomes or be underpowered to detect effects on clinically important outcomes. ${ }^{13}$ A number of additional studies have been completed evaluating the efficacy, safety and effectiveness of PMX-HP, including a larger multicentre RCT. ${ }^{14}{ }^{15}$ Accordingly, we propose to perform an up-to-date systematic review and evidence synthesis evaluating the impact of PMX-HP as an adjuvant therapy for critically ill patients with sepsis or septic shock on clinical outcomes and health services usage.

\section{Objectives}

To assess efficacy, safety and effectiveness of PMX-HP for adult patients with sepsis or septic shock.

\section{METHODS AND ANALYSIS}

Study design

We will perform a systematic review and meta-analysis, using the guidelines from the Cochrane Collaboration and Centre for Reviews and Dissemination, and described according to the PRISMA-P guideline (see online supplementary appendix 1 ).

\section{Study registration}

The systematic review protocol has been registered with the PROSPERO International Prospective Register of Systematic Reviews (http://www.crd.york.ac.uk/ prospero), and will be reported using Preferred Reporting Items for Systematic review and Meta-Analysis protocols. ${ }^{16}$ Registration number CRD42016038356.

\section{Criteria for considering studies for this review Types of studies}

All relevant randomised controlled trials that investigate the effect of PMX-HP for patients with sepsis or septic shock will be included. We will also include clusterrandomised trials if the intracluster correlation coefficient is reported. We will exclude crossover studies because of the nature of sepsis requiring intensive treatment within a short period. We will also exclude quasi-randomised studies. We will include studies reported as full text, those published as an abstract only and unpublished data.

\section{Types of participants}

Adults aged 18 years or older with sepsis or septic shock will be included. Studies that included a minority $(<10 \%)$ of patients under 18 years, or studies with median or mean of age of patients over 20 years will be included.

The diagnosis of sepsis or septic shock will be based on the diagnosis with documented or clinically suspected systemic infection. Septic shock will be classically defined as a hypotension resistant to fluid administration requiring norepinephrine or other vasopressor. ${ }^{17}$ Patients who developed sepsis or septic shock after surgery will also be included.

Participants of any gender and ethnicity and who are treated in any setting will be included.

\section{Types of interventions}

The interventions will be the use of the PMX-HP fibre column for the treatment of sepsis or septic shock. Comparison will be performed between the patients who receive standard treatment plus PMX-HP (PMX-HP group) and the patients with standard treatment only or sham haemoperfusion (Control group).

\section{Types of outcome measures}

Primary outcomes:

1. 28-day all-cause mortality

2. Serious adverse events, defined as hypotension or massive bleeding

3. Changes in organ dysfunction scores (Changes in Sequential Organ Failure Assessment (SOFA) score over 72 hours after the treatment)

Secondary outcomes:

1. 90-day all-cause mortality

2. Changes in mean arterial blood pressure over 72 hours after the treatment 
3. Changes in endotoxin levels over 72 hours after the treatment

4. Duration of vasopressor therapy or 28-day vasopressor-free days

5. Duration of RRT

6. ICU length of stay

7. Cost related to health services

\section{Search methods for identification of studies}

PROSPERO was searched for any registered systematic reviews on this topic (26 May 2016).

\section{Electronic searches}

The search strategy was developed in consultation with a research librarian at the Alberta Research Centre for Health Evidence (ARCHE) at the University of Alberta and underwent peer review by another research librarian. ${ }^{18} \mathrm{~A}$ draft of the search strategy is available in online supplementary appendix 2 . We will obtain all relevant studies irrespective of language or publication status (published, unpublished, in press and in progress) from the following databases: MEDLINE (Ovid), EMBASE (Ovid), Cochrane Library, Health Technology Assessment Database (HTA), Cumulative Index to Nursing and Allied Health Literature (CINAHL), PubMed and 'Igaku Chuo Zasshi (ICHUSHI)'. ICHUSHI is a bibliographic database that was established in 1903 and is being updated by the Japan Medical Abstracts Society, a non-profit and nongovernmental body (http://www.jamas.or.jp/about/ english.htm). ICHUSHI contains bibliographic citations and abstracts from more than 2500 biomedical journals and other serial publications published in Japan. We will modify the MEDLINE search strategy for searching all the other databases. For ongoing trials, we will search the National Institute of Health Clinical Trials Register (https://clinicaltrials.gov/), the WHO International Clinical Trials Registry Platform (http://www.who.int/ ictrp/en/) and the University Hospital Medical Information Network Clinical Trials Registry (http:// www.umin.ac.jp/ctr/).

\section{Searching other resources}

We will manually search citations from all included studies. We will contact authors of the identified trials if necessary to inquire about unpublished studies. We will also contact experts in the field and selected industries that develop or license PMX-HP to identify additional unpublished and ongoing trials. If necessary, we will also search conference proceedings and manufacturer websites.

\section{Data collection and analysis}

\section{Selection of studies}

Two review authors ( $\mathrm{TF}$ and $\mathrm{RG}$ ) will independently screen titles and abstracts of all studies we identified by the search and code them as 'retrieve' (eligible or potentially eligible/unclear) or 'do not retrieve'. We will retrieve their full texts and identify studies for inclusion and record reasons for exclusion of the ineligible studies. We will resolve any disagreement through discussion or, if required, we will consult a third person (YK or $\mathrm{SMB})$. Agreement between the two review authors in determining study eligibility will be reported as percentage agreement and weighted $\kappa$. We will identify and exclude duplicates of the same study so that each study rather than each report is the unit of interest in the review.

\section{Data extraction and management}

We will use a standardised data extraction form for study characteristics and outcome data that has been piloted on at least one study in the review. Two review authors (TF and RG) will extract data from the included studies independently. Agreement between the two reviewers concerning the primary outcomes will be reported as percentage agreement and intraclass correlation coefficients. Agreement between the two reviewers in the risk of bias items will be reported as percentage agreement and weighted $\kappa$. Any disagreement will be resolved through discussion, or discussed with a third person (YK or SMB) if necessary. We will abstract the following information: study characteristics, patient characteristics, sample size, interventions, comparators, potential biases in the conduct of the trial, outcomes including adverse events, methods of statistical analysis, clinical trial registration and funding support.

Literature search results will be uploaded to Rayyan Software (https://rayyan.qcri.org/), an internet-based software program that facilitates collaboration among reviewers to select with titles and abstracts. Prior to the formal screening process, a calibration exercise will be undertaken to pilot and refine the screening sheet. Analyses will be performed using Review Manager Software (V.5.3, Cochrane Collaboration, http://tech. cochrane.org/RevMan) and GRADEpro GDT, http:// gradepro.org/.

\section{Assessment of risk of bias in included studies}

At least two reviewers will independently assess the risk of bias of the included studies using the tool described in the Cochrane Handbook for Systematic Reviews of Interventions. ${ }^{19}$ The domains listed in box 1 will be assessed.

The risk of bias, in each domain and overall, will be assessed as shown in table 1 . When inadequate details of randomisation and other characteristics of trials are provided, the risk of bias will be classified as unclear unless further information can be obtained by contacting the authors. If the assessors disagree, the final rating will be made by discussion or with the involvement of another member of the review group, if necessary. A study will be classified as being at low risk of attrition bias when data for all randomised patients are available. In the case of dropouts, a study may still be assessed as being at low risk of attrition bias when: 


\section{Box 1 Domains for assessment of the risk of bias}

1. Random sequence generation (selection bias).

2. Allocation concealment (selection bias).

3. Blinding of participants and personnel (performance bias).

4. Blinding of outcome assessment (assessment bias).

5. Incomplete outcome data reporting (attrition bias).

6. Selective outcome reporting (reporting bias).

7. Sponsorship bias.

8. Other biases (Cointervention imbalance).

- Missing outcome data are few and balanced in numbers across intervention groups, with similar reasons for missing data across groups;

- Reasons for missing outcome data are unlikely to be related to true outcome;

- Missing data have been imputed using appropriate methods.

Whenever possible, study protocols will be retrieved in order to assess the risk of reporting bias. A study will be considered to be at low risk of reporting bias when the study protocol is available and all of the study's prespecified outcomes that are of interest in the review have been reported in the prespecified way. When the study protocol is not available, the study will be classified as being at unclear risk of reporting bias unless reported information is sufficient to make a judgement.

\section{Measures of treatment effect \\ Dichotomous outcomes}

As the measure of treatment effect for dichotomous outcomes, we will use the RR and its 95\% CI because of its favourable mathematical properties. Graphical displays for meta-analysis performed on ratio scales will use a $\log$ scale.

\section{Continuous outcomes}

The unit of measurement to be used in the included studies will be identical. Data will therefore be pooled by calculating the mean difference (MD) with $95 \%$ CI.

\section{Unit of analysis issues}

Crossover trials

Clinical trials with a crossover design will be excluded in this review.

\begin{tabular}{ll}
$\begin{array}{l}\text { Table } 1 \\
\text { The risk } \\
\text { of bias }\end{array}$ & Essessment of the risk of bias \\
\hline Low risk & $\begin{array}{l}\text { Plausible bias unlikely to seriously alter the } \\
\text { results }\end{array}$ \\
Unclear risk & $\begin{array}{l}\text { Insufficient information to determine whether } \\
\text { the risk of bias is low or high }\end{array}$ \\
High risk & $\begin{array}{l}\text { Plausible bias that seriously weakens } \\
\text { confidence in the results }\end{array}$ \\
\hline
\end{tabular}

\section{Cluster-randomised trials}

Cluster-randomised trials will be included in this review as long as proper adjustment for the intracluster correlation is conducted. ${ }^{19}$ We will reduce the size of each trial to its effective sample size. A common design effect will be assumed across intervention groups. For dichotomous data, both the number of participants and the number experiencing the event should be divided by the same design effect. For continuous data, only the sample size needs to be reduced; means and SDs should remain unchanged.

\section{Multiple intervention groups}

Where multiple trial arms are reported in a single trial, arms will be combined as long as they can be regarded as subtypes of the same haemoperfusion therapy. When arms cannot be regarded as if in each of them a different subtype of the same intervention is administered, we will include only the relevant arms. In such a case, a single pairwise comparison will be used to avoid a unit of analysis error. For dichotomous outcomes, data from a different dosage of the same relevant active intervention arms will be summed into a single arm for comparison. For continuous outcomes, means and SDs will be combined. $^{19}$

\section{Dealing with missing data}

We will try to contact the study authors for all relevant missing data.

\section{Missing participants \\ Dichotomous outcomes}

The proportion of treatment failure will be calculated using an intention-to-treat following the principle 'once randomised always analysed'. To this end, all randomised patients for whom outcome data are not available will be assumed as treatment failures. Any assumptions and imputations to handle missing data will be clearly stated, and the effect of imputation will be explored by sensitivity analyses.

\section{Continuous outcomes}

An available cases analysis will be performed for patients with a final assessment presented by the original authors.

\section{Missing statistics}

When only $\mathrm{p}$ values or SE values are reported, we will calculate SDs according to Altman. ${ }^{20}$ If none of these values are available, and in the absence of online supplementary data after requests to the authors, the SDs will be calculated from CIs, t values or $\mathrm{p}$ values; ${ }^{19}$ or they will be imputed from other studies in the meta-analysis according to a validated method. ${ }^{21}$ We will examine the validity of these imputations in a sensitivity analysis. Where actual $\mathrm{p}$ values obtained from t-tests are reported, the corresponding $\mathrm{t}$ value will be obtained from a table of the t distribution. 


\section{Assessment of heterogeneity}

We will assess overall heterogeneity by visual inspection of the forest plots. We will also assess statistical heterogeneity using the $\mathrm{I}^{2}$ statistic (on a scale of $0 \%$ to $100 \%$ ) and $\chi^{2}$ test. $\mathrm{I}^{2}$ values above $50 \%$ will be considered to represent substantial statistical heterogeneity and be explored further. However, the importance of the observed $\mathrm{I}^{2}$ depends on the magnitude and direction of treatment effects and the strength of evidence for heterogeneity. ${ }^{19}$ Since the $\chi^{2}$ test has low power when studies have a small sample size or are few in number, we will use a $p$ value of 0.10 to determine statistical significance. To provide an indication of the spread of true investigation effects, we will also report between-study variance in a random-effects meta-analysis using $\tau^{2}$. For absolute measures of effect, an approximate $95 \%$ range of underlying effects can be obtained by creating an interval from $1.96 \times \tau$ below the random-effects pooled estimate, to $1.96 \times \tau$ above it.

\section{Assessment of reporting biases}

We will undertake comprehensive searches of multiple sources (including trial registries), increasing efforts to identify the unpublished materials and including non-English language publications as far as possible, to minimise the impact of reporting biases. We will also try to identify outcome reporting bias in trials by recording all trial outcomes, planned and reported, and noting where outcomes were missing. When we find evidence of missing outcomes, we will attempt to obtain any available data directly from the authors. Where this is not possible, and the missing data are thought to introduce serious bias, the impact of including such studies in the overall assessment of results will be explored by a sensitivity analysis. Funnel plots will be constructed, and visual inspection will be performed to investigate the asymmetry. When 10 or more trials are pooled for primary outcomes, tests for funnel plot asymmetry will be used to investigate the potential influence of reporting biases and small-study effects.

\section{Data synthesis}

Meta-analyses will be performed should a sufficient number of studies (three or more) are found that share study design and measurement of comparators; otherwise, we will describe the results from each studies. Given the likelihood of differing underlining disorders in the population of interest, such as abdominal sepsis and others, a random-effects model will be used in all analyses. $^{22}$

\section{Subgroup analysis and investigation of heterogeneity}

We expect considerable heterogeneity for the primary outcomes, and we will use subgroup and meta-regression analyses as exploratory tools to explain them. Exploring sources of heterogeneity may result in false-positive conclusions through multiple analyses. Since these analyses lack power, they are more likely to result in false-negative results. Giving thought to these limitations, we will perform the following a priori subgroup analyses for the participant group and intervention if sufficient detail is present in the eligible studies.

\section{Participants}

- Abdominal sepsis versus sepsis with other aetiologies

- Culture positive sepsis versus others

- Confirmed gram-negative sepsis versus others

- Patients with high-level endotoxin activity defined as endotoxin activity assay $\geq 0.6$ vs low-level endotoxin activity

- Surgical patients versus medical patients

- Sepsis versus septic shock

- Patients with acute kidney injury (AKI) versus others We will use the formal statistical test for heterogeneity across subgroup based on a random-effects model to test for subgroup interaction.

\section{Intervention}

- Single session versus two sessions versus more than two sessions

- Less than 2 hours versus 2 hours versus longer than 2 hours

\section{Sensitivity analysis}

To determine the sensitivity of the findings to the way in which we have conducted the analysis, we will perform sensitivity analyses in the following areas.

1. Risk of bias: We will include only trials with a low risk of bias in allocation concealment.

2. Imputed missing data: We will exclude trials for which missing data are imputed.

3. Statistical method of data synthesis: a random-effects model versus fixed-effect model.

\section{Summary of findings tables}

We will assess the quality of the body of evidence using the Grading of Recommendations Assessment, Development and Evaluation (GRADE). ${ }^{23}$ We will present the results of the review in the 'Summary of Findings' tables including the following outcomes: 28-day all-cause mortality, serious adverse events, changes in organ dysfunction scores, 90-day all-cause mortality, changes in mean arterial blood pressure over 72 hours after the treatment, duration of vasopressor therapy or vasopressor-free days, and cost related to health services.

\section{Ethics and dissemination}

The study will evaluate the clinical effect of PMX-HP for patients with sepsis/septic shock from available published and unpublished clinical trial data. Since no primary data are collected, formal health research ethics approval is not required. The study will be disseminated by peer-reviewed publication and conference presentation. If our protocol needs to be amended, the date, 
details of the change and the rationale will be documented in the revised protocol and updated on PROSPERO.

\section{DISCUSSION}

The role of endotoxin in sepsis is well established in the literature. $^{9}{ }^{10}$ PMX-HP was developed to remove and clear circulating endotoxin, ${ }^{2}{ }^{3}$ which leads to decreases in inflammatory cytokines and mediators. PMX-HP has also been reported to adsorb activated neutrophils ${ }^{24}$ and monocytes $^{25}$ in patients with sepsis. A variety of small open-label clinical trials have been published with generally promising results. ${ }^{13}$ Nevertheless, data from previous studies should be considered as inconclusive, as those trials inherit a high risk of bias, that is, underpowered or unblinded. The purpose of our planned systematic review is to determine efficacy, safety and effectiveness of PMX-HP for adult patients with sepsis or septic shock. The key strength of this protocol is its comprehensive search for relevant studies, including ongoing trials ${ }^{14}$ and unpublished data. No language restriction will be placed and a thorough search in the databases in the country where PMX-HP was developed will enable a data comprehensive review to update our knowledge.

There are several expected limitations for this review. First, we defined sepsis as documented or clinically suspected systemic infection and septic shock as sepsis-induced hypotension persisting despite fluid resuscitation, in line with the well-known definition. ${ }^{17}$ A new definition for sepsis was proposed recently, ${ }^{26}$ and future research on sepsis might use this new definition. To address the issue of variation in participants in interpreting and applying the results, we propose to perform a subgroup analyses for the participants according to endotoxin activity levels. Second, the results of this review will depend on the quality of the studies we identify, which might be low as in the previous review. ${ }^{13}$ We will, therefore, perform sensitivity analyses to see whether the findings will change depending on the risk of bias of each study. Finally, small studies with high heterogeneity could make it difficult to interpret the obtained results.

However, we expect the present review to provide the most comprehensive and up-to-date critical summary of available evidence regarding the use of PMX-HP for sepsis or septic shock in the ICU and will help guide treatment recommendations of sepsis or septic shock in the clinical practice guidelines. ${ }^{5}$

\footnotetext{
Author affiliations

${ }^{1}$ Department of Epidemiology and Preventive Service, Kyoto University Graduate School of Medicine, Kyoto, Japan

${ }^{2}$ Research Fellow of Japan Society for the Promotion of Science

${ }^{3}$ Department of Surgery, Kyoto University Hospital, Kyoto, Japan

${ }^{4}$ Department of Respiratory Medicine, Hyogo Prefectural Amagasaki General

Medical Center, Amagasaki, Japan

${ }^{5}$ Department of Pediatrics, Alberta Research Center for Health Evidence

(ARCHE), University of Alberta, Edmonton, Alberta, Canada

${ }^{6}$ Department of Critical Care Medicine, Faculty of Medicine and Dentistry, University of Alberta, Edmonton, Alberta, Canada

${ }^{7}$ Department of Health Promotion and Human Behavior, Kyoto University Graduate School of Medicine, Kyoto, Japan
}

Acknowledgements The authors thank Dr Toshiyuki Suwa at Osaka University for his assistance with the search strategy design in Japanese databases, and Ms. Tara Landry at Montreal General Hospital, McGill University Health Centre for her peer reviewing the MEDLINE search strategy.

Contributors TAF is the guarantor of the protocol. TF and SMB conceived the project. TF and RG worked equally on the initial architecture for the review with methodological input from YK, RF and SMB. TAF helped draft the article and revise it critically for important intellectual content. All authors gave final approval of the version to be published.

Funding TF is supported by the Japan Society for the Promotion of Science. SMB is supported by a Canada Research Chair in Critical Care Nephrology and is a Steering Committee member of the EUPHRATES trial. TAF has received lecture fees from Eli Lilly, Janssen, Meiji, MSD, Otsuka, Pfizer and Tanabe-Mitsubishi, and consultancy fees from Sekisui Chemicals and Takeda Science Foundation. He has received royalties from Igaku-Shoin and Nihon Bunka Kagaku-sha publishers. He has received grant or research support from the Japanese Ministry of Education, Science and Technology, the Japanese Ministry of Health, Labour and Welfare, the Japan Society for the Promotion of Science, the Japan Foundation for Neuroscience and Mental Health, Mochida and Tanabe-Mitsubishi. He is diplomate of the Academy of Cognitive Therapy. This review will be supported in part by JSPS KAKENHI (Grant-in-Aid for Scientific Research) grant number 26670314 to TAF. Only the named authors have ultimate authority over these activities.

\section{Competing interests None declared.}

Provenance and peer review Not commissioned; externally peer reviewed.

Open Access This is an Open Access article distributed in accordance with the Creative Commons Attribution Non Commercial (CC BY-NC 4.0) license, which permits others to distribute, remix, adapt, build upon this work noncommercially, and license their derivative works on different terms, provided the original work is properly cited and the use is non-commercial. See: http:// creativecommons.org/licenses/by-nc/4.0/

\section{REFERENCES}

1. Kaukonen KM, Bailey M, Suzuki S, et al. Mortality related to severe sepsis and septic shock among critically ill patients in Australia and New Zealand, 2000-2012. JAMA 2014;311:1308-16.

2. Gaieski DF, Edwards JM, Kallan MJ, et al. Benchmarking the incidence and mortality of severe sepsis in the United States. Crit Care Med 2013;41:1167-74.

3. Suffredini AF, Munford RS. Novel therapies for septic shock over the past 4 decades. JAMA 2011;306:194-9.

4. Dellinger RP, Carlet JM, Masur $\mathrm{H}$, et al. Surviving Sepsis Campaign Management Guidelines Committee. Surviving Sepsis Campaign guidelines for management of severe sepsis and septic shock. Crit Care Med 2004;32:858-73.

5. Dellinger RP, Levy MM, Rhodes A, et al. Surviving Sepsis Campaign Guidelines Committee including the Pediatric Subgroup. Surviving sepsis campaign: international guidelines for management of severe sepsis and septic shock: 2012. Crit Care Med 2013:41:580-637.

6. Marshall JC, Foster D, Vincent JL, et al. MEDIC study. Diagnostic and prognostic implications of endotoxemia in critical illness: results of the MEDIC study. J Infect Dis 2004;190:527-34.

7. Shoji H, Tani T, Hanasawa K, et al. Extracorporeal endotoxin removal by polymyxin $\mathrm{B}$ immobilized fiber cartridge: designing and antiendotoxin efficacy in the clinical application. Ther Apher 1998;2:3-12.

8. Shoji H. Extracorporeal endotoxin removal for the treatment of sepsis: endotoxin adsorption cartridge (Toraymyxin). Ther Apher Dial 2003;7:108-14.

9. Danner RL, Elin RJ, Hosseini JM, et al. Endotoxemia in human septic shock. Chest 1991;99:169-75.

10. Casey LC, Balk RA, Bone RC. Plasma cytokine and endotoxin levels correlate with survival in patients with the sepsis syndrome. Ann Intern Med 1993;119:771-8.

11. Vincent JL, Laterre PF, Cohen J, et al. A pilot-controlled study of a polymyxin B-immobilized hemoperfusion cartridge in patients with severe sepsis secondary to intra-abdominal infection. Shock 2005;23:400-5.

12. Cruz DN, Antonelli M, Fumagalli R, et al. Early use of polymyxin B hemoperfusion in abdominal septic shock: the EUPHAS randomized controlled trial. JAMA 2009;301:2445-52. 
13. Cruz DN, Perazella MA, Bellomo R, et al. Effectiveness of polymyxin B-immobilized fiber column in sepsis: a systematic review. Crit Care 2007;11:R47.

14. Klein DJ, Foster D, Schorr CA, et al. The EUPHRATES trial (Evaluating the Use of Polymyxin B Hemoperfusion in a Randomized controlled trial of Adults Treated for Endotoxemia and Septic shock): study protocol for a randomized controlled trial. Trials 2014; $15: 218$.

15. Payen DM, Guilhot J, Launey $Y$, et al. ABDOMIX Group. Early use of polymyxin B hemoperfusion in patients with septic shock due to peritonitis: a multicenter randomized control trial. Intensive Care Med 2015;41:975-84.

16. Shamseer L, Moher D, Clarke M, et al. PRISMA-P Group. Preferred reporting items for systematic review and meta-analysis protocols (PRISMA-P) 2015: elaboration and explanation. BMJ 2015;349: g7647.

17. Levy MM, Fink MP, Marshall JC, et al. International Sepsis Definitions Conference. 2001 SCCM/ESICM/ACCP/ATS/SIS international sepsis definitions conference. Intensive Care Med 2003;29:530-8.

18. Sampson M, McGowana J, Cogo E, et al. An evidence-based practice guideline for the peer review of electronic search strategies. $J$ Clin Epidemiol 2009;62:944-52.
19. Higgins JPT, Green S, the Cochrane Collaoration. Cochrane handbook for systematic reviews of interventions. Chichester, UK: John Wiley \& Sons, 2008.

20. Altman DG, Bland JM. Detecting skewness from summary information. BMJ 1996;313:1200.

21. Furukawa TA, Barbui C, Cipriani A, et al. Imputing missing standard deviations in meta-analyses can provide accurate results. J Clin Epidemiol 2006;59:7-10.

22. DerSimonian R, Laird N. Meta-analysis in clinical trials. Control Clin Trials 1986;7:177-88.

23. Guyatt G, Oxman AD, Akl EA, et al. GRADE guidelines: 1. Introduction-GRADE evidence profiles and summary of findings tables. J Clin Epidemiol 2011;64:383-94.

24. Kumagai T, Takeyama N, Yabuki T, et al. Apheresis of activated leukocytes with an immobilized polymyxin B filter in patients with septic shock. Shock 2010;34:461-6.

25. Tsujimoto $\mathrm{H}$, Ono $\mathrm{S}$, Hiraki $\mathrm{S}$, et al. Hemoperfusion with polymyxin B-immobilized fibers reduced the number of CD16+CD14+ monocytes in patients with septic shock. J Endotoxin Res 2004; $10: 229-37$.

26. Singer M, Deutschman CS, Seymour CW, et al. The Third International Consensus Definitions for Sepsis and Septic Shock (Sepsis-3). JAMA 2016;315:801-10. 